\title{
Estado nutricional de portadoras de síndrome dos ovários policísticos segundo os diferentes índices antropométricos
}

\author{
Nutritional status of carriers of polycystic ovarian syndrome according to different anthropometric \\ indices
}

DOI: $10.37111 /$ braspenj.2020354011

Joene Vitória Rocha Santos

Bruna Yhang da Costa Silva²

\section{Unitermos:}

Síndrome do Ovário Policístico. Antropometria. Estado Nutricional.

\section{Keywords:}

Polycystic Ovary Syndrome. Anthropometry. Nutritional status.

\section{Endereço para correspondência: \\ Bruna Yhang da Costa Silva}

Rua Estevão Remígio de Freitas, 1145 - Monsenhor Otávio - Limoeiro do Norte, CE, Brasil - CEP 62930 000

E-mail: bruna.yhang@ifce.edu.br

Submissão:

14 de maio de 2020

Aceito para publicação

12 de dezembro de 2020

\section{RESUMO}

Introdução: Mulheres portadoras de síndrome dos ovários policísticos apresentam em comum várias alterações hormonais e metabólicas, dentre as quais se destaca a resistência periférica à insulina, a qual manifesta-se mais frequentemente naquelas com sobrepeso ou obesidade. O objetivo deste estudo foi avaliar o estado nutricional de portadoras de síndrome dos ovários policísticos e correlacionar os índices antropométricos recentemente propostos com os tradicionais. Método: A pesquisa teve abordagem quantitativa, transversal e analítica. Os participantes foram 40 mulheres, na faixa etária de 20 a 59 anos, do município de Jaguaruana - Ceará. Foi aplicado um questionário, a fim de obter informações sobre história socioeconômica, cultural, história clínica, história da doença atual e revisão dos sistemas corporais pertinentes à doença. Para avaliação antropométrica utilizou-se o índice de massa corpórea (IMC), circunferência da cintura (CC), relação cintura-quadril (RCQ), índice de adiposidade corporal, body shape index (ABSI), que é um indicador de mortalidade prematura e do excesso de adiposidade abdominal e body roundness index (BRI), que é um ótimo preditor da porcentagem de gordura corporal e de tecido adiposo visceral. Resultados: Ao avaliar o IMC, viu-se que a eutrofia foi a categoria predominante $(40 \%)$. Porém, $55 \%$ das participantes apresentaram algum grau de excesso de peso (pré-obesidade ou obesidade). A maioria das participantes não apresentou risco para doenças cardiovasculares em relação aos diagnósticos de CC e RCQ. Quanto aos novos índices, inferiuse que a forma corporal predominante na amostra é a levemente arredondada. Conclusões: Constatou-se que as participantes dentro dos maiores tercis de ABSI mostraram ter também maiores valores de RCQ. Já quanto ao BRI observou-se uma relação diretamente proporcional entre as médias de seus tercis e aquelas dos índices/medidas tradicionais. A associação entre os tradicionais e os índices/medidas antropométricos mais recentemente propostos possibilita uma melhor e mais completa interpretação do estado nutricional, em especial se realizadas de forma seriada.

\section{ABSTRACT}

Introduction: Women with polycystic ovary syndrome have several hormonal and metabolic changes in common, among which peripheral insulin resistance stands out, which manifests itself more frequently in those with overweight or obesity. The objective of this study was to evaluate the nutritional status of patients with polycystic ovary syndrome and to correlate the most recent anthropometric indexes proposed with traditional ones. Methods: The research had a quantitative, transversal and analytical approach. The participants were 40 women, aged 20 to 59 years, from the city of Jaguaruana - Ceará. A questionnaire was applied in order to obtain information about cultural and socioeconomic history, clinical history, history of the current disease and revision of the body systems pertinent to the disease. The body mass index (BMI), waist circumference (WC), waist-hip ratio (WHR), body adiposity index (ABSI), body shape index and body shape index, which is an indicator of premature mortality and excess abdominal adiposity and body roundness index (BRI), which is a great predictor of the percentage of body fat and visceral adipose tissue. Results: When assessing BMI, it was seen that eutrophy was the predominant category $(40 \%)$. However, $55 \%$ of the participants had some degree of excess weight (pre-obesity or obesity). Most participants were not at risk for cardiovascular disease in relation to the diagnoses of WC and WHR. As for the new indices, it was inferred that the predominant body shape in the sample is slightly rounded. Conclusions: It was found that the participants within the largest $A B S I$ tertiles were also shown to have higher WHR values. As for the BRI, a directly proportional relationship was observed among the averages of its tertiles and those of the traditional indices/measures. The association between traditional and the most recently proposed anthropometric indices/measures allows for a better and more complete interpretation of nutritional status, especially if performed in a serial manner.

1. Nutricionista, especialista, Instituto Federal de Ciência e Tecnologia do Ceará - campus Limoeiro do Norte - Mestrado Acadêmico em Tecnologia de Alimentos, Limoeiro do Norte, CE, Brasil.

2. Nutricionista, professora doutora, Instituto Federal de Ciência e Tecnologia do Ceará - campus Limoeiro do Norte - Curso de Bacharelado em Nutrição, Limoeiro do Norte, CE, Brasil. 


\section{INTRODUÇÃO}

A síndrome dos ovários policísticos (SOP) é uma heterogênea, multifatorial e complexa endocrinopatia, caracterizada pela anovulação crônica, hiperandrogenismo clínico ou bioquímico e/ou ovários policísticos detectados por ultrassom, que afeta aproximadamente $6 \%$ a $20 \%$ de mulheres em idade reprodutiva. Esta síndrome é multigênica e na sua etiologia inclui alterações na biossíntese, regulação e ação dos andrógenos, na ação e secreção de insulina, na ação e secreção das gonadotrofinas e na síntese e metabolismo do ácido retinoico ${ }^{1,2}$.

Há uma série de comorbidades associadas à SOP, de modo que as mulheres que são portadoras apresentam uma maior prevalência de sobrepeso ou obesidade, resistência à ação da insulina, dislipidemia, hipertensão, diabetes e marcadores precoces de aterosclerose. Mais recentemente, a SOP tem sido associada também ao aparecimento de transtornos psicológicos, como ansiedade e depressão. Embora o fator central comum a estes distúrbios seja a resistência periférica à insulina, a literatura evidencia que as alterações metabólicas decorrentes da SOP se manifestam mais frequentemente em mulheres com sobrepeso ou obesidade ${ }^{3}$.

A obesidade é uma característica bastante comum, estando presente em cerca de $27 \%$ das mulheres com SOP, mas estima-se $46 \%$ dessas mulheres apresentam o índice de massa corporal (IMC) $>25 \mathrm{~kg} / \mathrm{m}^{2}$ e há, frequentemente, concentração de gordura abdominal (visceral). Como consequência, a circunferência da cintura (CC) está aumentada nesses casos $(>88 \mathrm{~cm})$, assim como a relação cintura-quadril (RCQ) $(>0,85)^{4}$.

Existem, também, índices mais atuais que são muito bem correlacionados com a resistência à ação da insulina e que podem ser usados como novos marcadores para avaliar a interferência desta síndrome no estado nutricional de suas portadoras e desse estado nutricional nas morbidades, caso seja inadequado ${ }^{4}$.

Deste modo, sabendo que mulheres com SOP têm a resistência à insulina como uma alteração endócrino-metabólica comum, que esta característica se acentua na presença de excesso de peso e, ainda, que da resistência à insulina e do excesso ponderal podem decorrer várias comorbidades, estudos que contribuem com a elucidação de estratégias práticas e eficientes de diagnóstico nutricional na rotina de acompanhamento dessas mulheres são fundamentais, para que elas convivam com a doença sem prejuízos significantes para a sua qualidade de vida.

Diante do exposto, este estudo teve como objetivo avaliar o estado nutricional de portadoras de SOP atendidas em uma Unidade Básica de Saúde (UBS) da cidade de Jaguaruana - Ceará, por meio de aplicação de anamnese nutricional e avaliação antropométrica e, ainda, correlacionar os índices antropométricos mais recentemente propostos com os tradicionais.

\section{MÉTODO}

Trata-se de um estudo quantitativo, transversal e analítico, realizado com mulheres portadoras de SOP, adultas, atendidas na UBS da Família Dr. Carlos Alberto de Araúio (Sede I), da cidade de Jaguaruana, Ceará, entre outubro e dezembro de 2016.

Participaram do estudo mulheres com diagnóstico confirmado de SOP realizado ou confirmado pela equipe médica da referida UBS, que se encontravam em acompanhamento médico, com idade entre 20 e 59 anos, isto é, adultas segundo a World Health Organization $(\mathrm{WHO})^{5}$ e que aceitaram participar mediante assinatura do Termo de Consentimento Livre e Esclarecido.

Foram excluídas gestantes ou mulheres com doenças que interferem no peso corporal, como: doenças da tireoide, doenças das glândulas adrenais (doença de Addison, síndrome de Cushing, feocromocitoma), hepatite, cirrose, AIDS, tuberculose e/ou neoplasias 6 . A amostra estimada foi de 68 mulheres, que corresponde a todas as portadoras de SOP contidas nos registros das agentes comunitárias de saúde da UBS. Contudo, somente 40 participaram do estudo devido à recusa das demais em responder aos questionamentos ou impossibilidade de contato.

A coleta de dados consistiu na aplicação de um formulário socioeconômico e cultural (idade, estado civil, escolaridade, renda e prática de exercícios físicos), de história clínica (abordando doenças associadas), história da doença atual e revisão dos sistemas corporais acometidos comumente pela SOP (gastrointestinal e reprodutor).

Para avaliar o estado nutricional, foram aferidas medidas de peso, altura, CC, circunferência do quadril (CQ) e circunferência abdominal (CA). Para mensuração do peso foi utilizada balança digital Plenna ${ }^{\circledR}$ calibrada, com graduação de $100 \mathrm{~g}$ e capacidade máxima de $150 \mathrm{~kg}$. Para a estatura utilizou-se estadiômetro portátil Sanny ${ }^{\circledR}$, de capacidade máxima de 2 metros. As circunferências foram aferidas com fita métrica inelástica Sanny ${ }^{\circledR}$.

A aferição destas medidas permitiu a categorização do IMC segundo a $\mathrm{WHO}^{5}$, avaliar o risco para doenças cardiovasculares segundo a CC, sendo que os pontos de corte adotados para CC foram: $\geq 80 \mathrm{~cm}$ é igual a risco aumentado para doenças cardiovasculares e $\geq 88 \mathrm{~cm}$ representa risco muito aumentado para os mesmos problemas. $R C Q$, que tem como ponto de corte favorável valores $<0,85$ e desfavorável $\geq 0,85$, também segundo a $\mathrm{WHO}^{7}$. Foram classificados também o índice de adiposidade corporal 
$(\mathrm{IAC})^{8}$, que é calculado com base na circunferência do quadril e altura e tem como pontos de corte os seguintes percentuais: $\leq 30$ (normal), $\geq 35$ (sobrepeso), $\geq 40$ (obesidade), e por fim, o body shape index $(A B S I)^{9}$ e o body roundness index $(\mathrm{BRI})^{10}$.

$\mathrm{O} A \mathrm{BS}$ I é calculado com base em peso, altura e circunferência da cintura. É um indicador de mortalidade prematura e do excesso de adiposidade abdominal que considera valores acima de 0,08 , que consiste na média de uma população saudável, como indicativo de excesso de adiposidade visceral, cujo cálculo é estabelecido por Krakaver e Krakaver ${ }^{11}$, mas vale ressaltar que, no período da construção deste estudo, este valor de ponto de corte $(0,08)$ não era claro, não sendo adotado como parâmetro de classificação. Sua fórmula está disposta abaixo:

$$
A B S I=\frac{C C}{(I M C)^{2 / 3} \cdot(P E S O)^{1 / 2}}
$$

Já o BRI é calculado com base na altura e circunferência abdominal e é um ótimo preditor da porcentagem de gordura corporal e de tecido adiposo visceral estabelecido por Thomas et al. ${ }^{10}$, o qual define que, quanto mais próximo de um (valor mínimo) for o resultado, mais fina é a forma corporal. Por outro lado, valores mais próximos de 16 (máximo) sugerem um corpo mais arredondado e, assim, maior risco de complicações cardiovasculares. Seu cálculo ocorre em duas etapas:

$$
\begin{aligned}
& \epsilon=\sqrt{1-\left(\frac{(C A /(2 \pi))^{2}}{(0,5 x \text { altura })^{2}}\right)} \\
& \mathrm{BRI}=364,2-(365,5 x \epsilon \epsilon)
\end{aligned}
$$

BRI e o ABSI das mulheres foram distribuídos em tercis e, em seguida, foram determinadas as médias e desvio padrão de cada tercil. Para os demais dados, os resultados foram apresentados em frequências absolutas e percentuais, médias e desvios-padrão (média $\pm \mathrm{dp}$ ).

Quanto à análise estatística, o teste de Shapiro Wilk foi utilizado para verificar a normalidade dos dados. A partir do teste não-paramétrico de Kruskal-Wallis (análise de variância) verificou-se se as médias dos índices/medidas tradicionais diferiram entre os tercis de ABSI e BRI. Adotou-se $p<0,05$ como significante. O software Statistical Package for the Social Sciences (SPSS) versão 20 foi utilizado como auxiliar.

Este estudo foi aprovado pelo Comitê de Ética em Pesquisa do Instituto Federal de Educação, Ciência e Tecnologia do Ceará, sob parecer 1.961.282, de acordo com a Resolução do Conselho Nacional de Saúde n 466/2012.

\section{RESULTADOS}

Dentre a população elegível para o estudo (68 participantes), participaram 40 mulheres, cujos perfis socioeconômico e clínico são demonstrados na Tabela 1. As entrevistadas apresentaram média de idade de 26,75 $\pm 6,11$ anos, variando de 20 a 43 anos, a maioria era solteira (70\%), com ensino médio $(57,5 \%)$ e renda mensal de até um salário mínimo (37,5\%).

\begin{tabular}{|c|c|c|}
\hline Variáveis & $\mathbf{N}$ & $\%$ \\
\hline \multicolumn{3}{|l|}{ Atividade física } \\
\hline Sim & 22 & 55 \\
\hline Não & 18 & 45 \\
\hline \multicolumn{3}{|l|}{ Doenças Associadas } \\
\hline Hipertensão arterial & 3 & 7,5 \\
\hline Gastrite & 2 & 5 \\
\hline Sinusite & 2 & 5 \\
\hline Fibromialgia & 1 & 2,5 \\
\hline Cálculos renais & 1 & 2,5 \\
\hline Artrite reumatoide & 1 & 2,5 \\
\hline Não possuem & 30 & 75 \\
\hline \multicolumn{3}{|l|}{ Alergias } \\
\hline $\operatorname{Sim}$ & 9 & 22,50 \\
\hline Não & 31 & 77,50 \\
\hline \multicolumn{3}{|l|}{ Tratamento da SOP } \\
\hline $\operatorname{Sim}$ & 18 & 45 \\
\hline Não & 22 & 55 \\
\hline \multicolumn{3}{|l|}{ Digestão } \\
\hline Sem queixas & 24 & 60 \\
\hline Com queixas & 16 & 40 \\
\hline \multicolumn{3}{|l|}{ Evacuação } \\
\hline Sem queixas & 25 & 62,50 \\
\hline Com queixas & 15 & 37,50 \\
\hline \multicolumn{3}{|l|}{ Menstruação } \\
\hline Sem queixas & 17 & 42,50 \\
\hline Com queixas & 23 & 57,50 \\
\hline \multicolumn{3}{|l|}{ Sinais de TPM } \\
\hline Sim & 34 & 85 \\
\hline Não & 6 & 15 \\
\hline \multicolumn{3}{|l|}{ Amenorreia } \\
\hline Sim & 22 & 55 \\
\hline Não & 18 & 45 \\
\hline \multicolumn{3}{|l|}{ Partos anteriores } \\
\hline Sim & 10 & 25 \\
\hline Não & 30 & 75 \\
\hline
\end{tabular}

Tabela 1 - Perfil clínico e de atividade física das portadoras de SOP, Jaguaruana-CE, 2017. 
A prática de atividade física foi referida por $55 \%$ delas, metade das quais a faziam duas a quatro vezes por semana e as demais, cinco vezes por semana a diariamente.

Acerca de comorbidades, 25\% das entrevistadas apresentavam algum tipo de afecção e $75 \%$ não apresentavam. As alergias estiveram presentes em $22,5 \%$ das participantes, sendo as mais referidas a medicamentos (10\%) e alimentos (7,5\%). Quanto ao tratamento para SOP, era utilizado por $45 \%$ das participantes, todas a partir do uso de anticoncepcionais.

Quando questionadas sobre a saúde digestiva, 60\% das participantes não se queixaram. Entre as demais predominaram pirose frequente (17,5\%), distensão abdominal (10\%), refluxo gástrico $(7,5 \%)$ e náuseas $(5 \%)$. Dentre as pacientes que alegaram queixas quanto à evacuação $(37,5 \%), 17,5 \%$ relataram dor ao evacuar, $12,5 \%$ necessidade de impor força no ato, $5 \%$ incontinência e 2,5\% flatulência.

Quanto à saúde do sistema reprodutor, $42,5 \%$ das mulheres negaram queixas clínicas durante o período menstrual. As demais apresentaram predomínio de cólicas $(42,5 \%)$, dores de cabeça (10\%) e nas pernas (5\%). A maioria das entrevistadas (85\%) referiu sinais de tensão pré-menstrual, dentre eles: alterações de humor $(67,5 \%)$, maior apetite $(10 \%)$ e irritação (7,5\%). Como é uma característica marcante da SOP, avaliou-se também a presença de amenorreia, referida por $55 \%$ das participantes, mas somente na ausência de anticoncepcional. Partos anteriores foram referidos por $25 \%$ das mulheres.

Voltando-se para a análise do estado nutricional, principal objetivo deste trabalho, a Tabela 2 expõe a categorização das portadoras de SOP, segundo os índices/medidas antropométricos.

Ao avaliar o IMC, viu-se que a maior porcentagem das participantes apresentou valor que corresponde à eutrofia, porém ao somar as porcentagens de pré-obesidade e obesidade percebe-se que $55 \%$ das mulheres apresentaram algum grau de excesso de peso.

A CC sugeriu que a maior parte do grupo não apresentava excessiva deposição de gordura abdominal $(57,5 \%)$, mas a média do grupo foi de $80,24 \pm 12,37 \mathrm{~cm}$, que corresponde a risco elevado para morbidades associadas a complicações metabólicas (encontradas em 10\% das mulheres).

Para avaliar a propensão para doenças cardiovasculares (DCV), investigou-se também a $R C Q$, que mostrou predominância de mulheres sem risco $(82,5 \%)$. O mesmo foi observado para a média do grupo $(0,77 \pm 0,14)$. Analisando - IAC, observa-se que $50 \%$ das participantes apresentava um índice de adiposidade normal (50\%). As 50\% restantes apresentavam algum grau de excesso de gordura corporal ( $30 \%$ com sobrepeso e $20 \%$ com obesidade). A média de IAC $(33,64 \pm 7,42 \%)$ categoriza o grupo na faixa de sobrepeso.
Tabela 2 - Categorização das portadoras de SOP segundo os índices/ medidas antropométricos, Jaguaruana-CE, 2017.

\begin{tabular}{|c|c|c|c|}
\hline Diagnóstico & $\mathbf{N}$ & $\%$ & Média ( \pm dp) \\
\hline \multicolumn{4}{|l|}{ IMC } \\
\hline Moderadamente abaixo & 2 & 5 & $16,75 \pm 0,10$ \\
\hline Normal & 16 & 40 & $22,42 \pm 1,06$ \\
\hline Pré-obesidade & 12 & 30 & $26,91 \pm 1,53$ \\
\hline Obesidade grau I & 7 & 17,50 & $32,25 \pm 1,48$ \\
\hline Obesidade grau II & 2 & 5 & $36,19 \pm 0,20$ \\
\hline Obesidade grau III & 1 & 2,50 & $42,68 \pm 0$ \\
\hline \multicolumn{4}{|l|}{ Circunferência da Cintura } \\
\hline Sem risco & 23 & 57,50 & $71,40 \pm 5,65$ \\
\hline Risco elevado & 4 & 10 & $83,30 \pm 2,89$ \\
\hline Risco muito elevado & 13 & 32,50 & $98,80 \pm 7,66$ \\
\hline \multicolumn{4}{|l|}{ Relação Cintura-Quadril } \\
\hline Sem riscos para DCV & 33 & 82,50 & $0,75 \pm 0,06$ \\
\hline Com riscos para DCV & 7 & 17,50 & $0,89 \pm 0,04$ \\
\hline \multicolumn{4}{|c|}{ Índice de Adiposidade Corporal } \\
\hline Normal & 20 & 50 & $29,35 \pm 2,35$ \\
\hline Sobrepeso & 12 & 30 & $35,16 \pm 1,46$ \\
\hline Obesidade & 8 & 20 & $42,08 \pm 2,62$ \\
\hline \multicolumn{4}{|l|}{ ABSI } \\
\hline Abaixo da média & 18 & 45 & $0,069 \pm 0,015$ \\
\hline Média & 3 & 7,50 & $0,073 \pm 0$ \\
\hline Acima da média & 19 & 47,50 & $0,076 \pm 0,002$ \\
\hline \multicolumn{4}{|l|}{ BRI } \\
\hline Abaixo da média & 38 & 95 & $4,60 \pm 1,70$ \\
\hline Acima da média & 2 & 5 & $9,83 \pm 0,56$ \\
\hline
\end{tabular}

$\mathrm{ABSI}$ = body shape index; $\mathrm{BRI}=$ body roundness index; $\mathrm{DCV}=$ doenças cardiovasculares; IMC = índice de massa corpórea.

Quanto aos novos índices (ABSI e BRI), seus valores médios foram de, respectivamente, 0,073 $\pm 0,004$ e 4,8 \pm 2,12 , sugerindo uma forma corporal levemente arredondada.

Analisando a descrição das médias de $A B S I$ e $B R I$ e os índices/medidas tradicionais (IMC, CC, RCQ, IAC), foram elaborados gráficos, que demonstram as médias destes últimos, conforme os tercis individuais dos dois primeiros (Figuras 1 a 6).

Pode-se observar que CC e a RCQ apresentaram um aumento de médias, de acordo com os tercis de ABSI. Em relação ao BRI, todos os índices/medidas tradicionais apresentaram este comportamento. Entretanto, quando analisado estatisticamente, estes resultados se confirmam para $A B S I$ versus $R C Q(p=0,02$; para $C C, p=0,288)$ e para BRI versus IMC, CC, RCQ e IAC (respectivamente, $p<0,001$, $p$ $<0,001, p=0,027$ e $p<0,001$ ). 
100

90

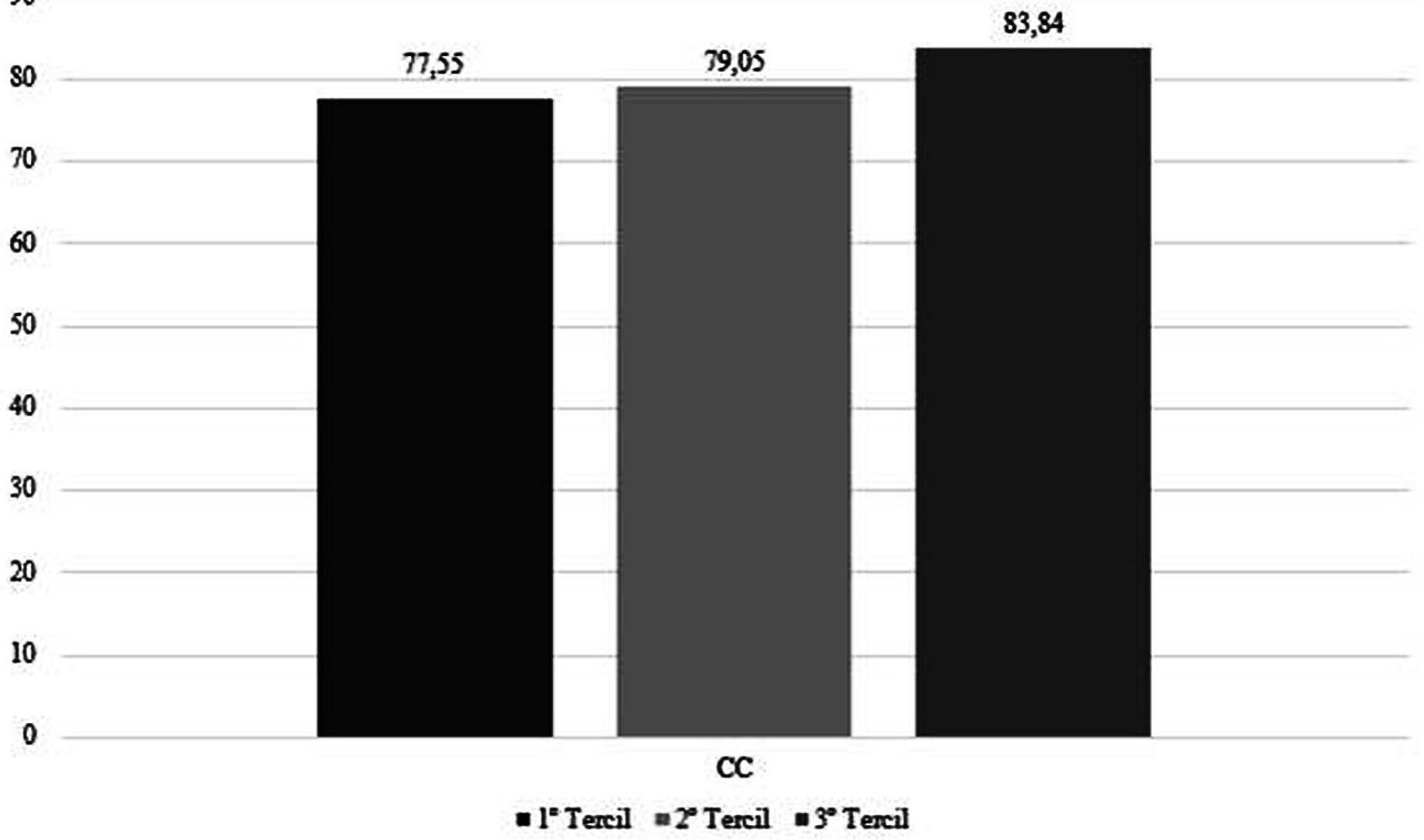

Figura 1 - Variação das médias de CC conforme tercis de ABSI, Jaguaruana-Ceará, 2017. ABSI = body shape index; CC = circunferência da cintura.

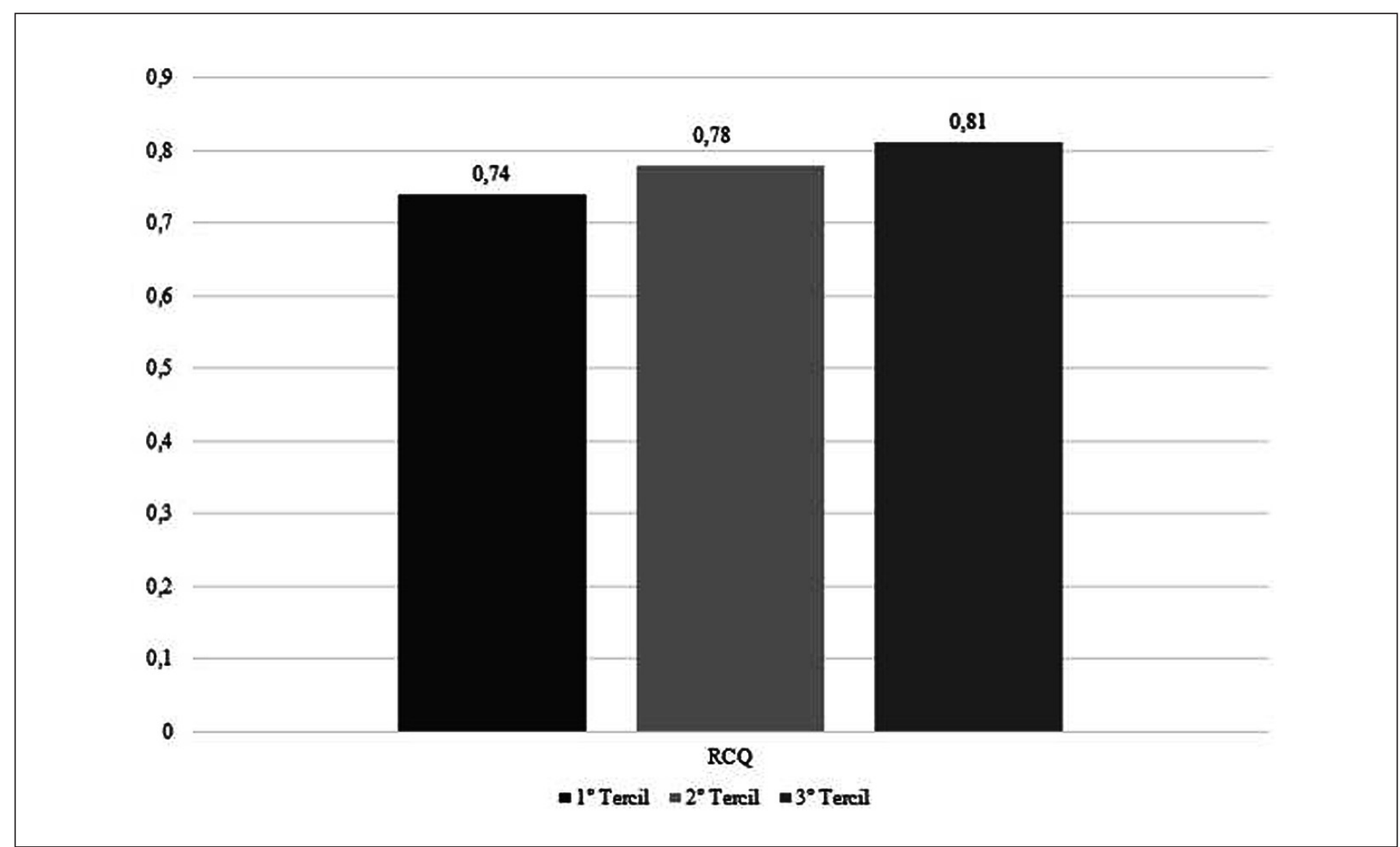

Figura 2 - Variação das médias de RCQ conforme tercis de ABSI, Jaguaruana-Ceará, 2017. ABSI = body shape index; $R C Q=$ relação cintura-quadril. 


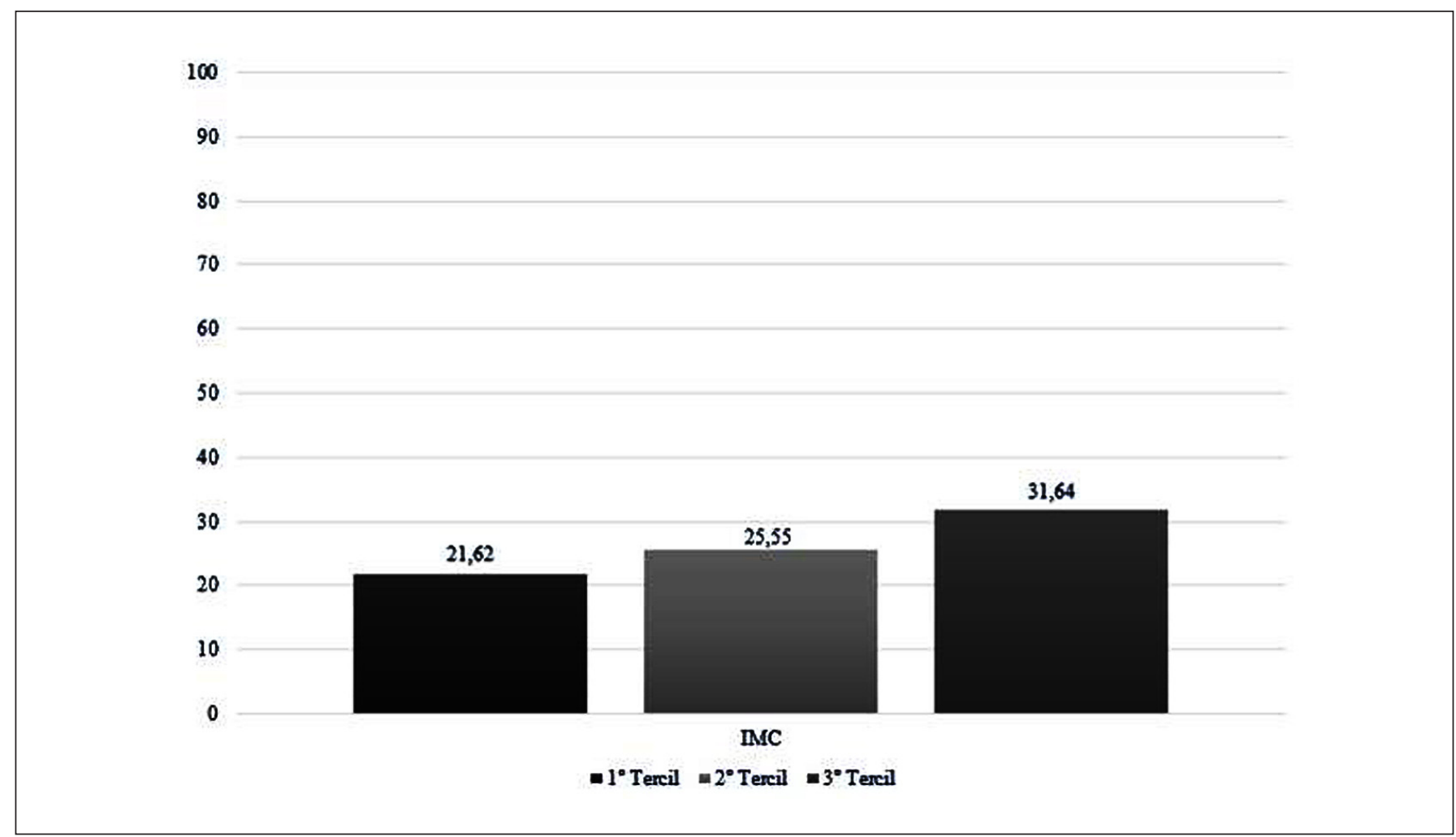

Figura 3 - Variação das médias do IMC $\left(\mathrm{kg} / \mathrm{m}^{2}\right)$ conforme tercis de BRI, Jaguaruana-Ceará, 2017. BRI = body roundness index; IMC = indice de massa corpórea.

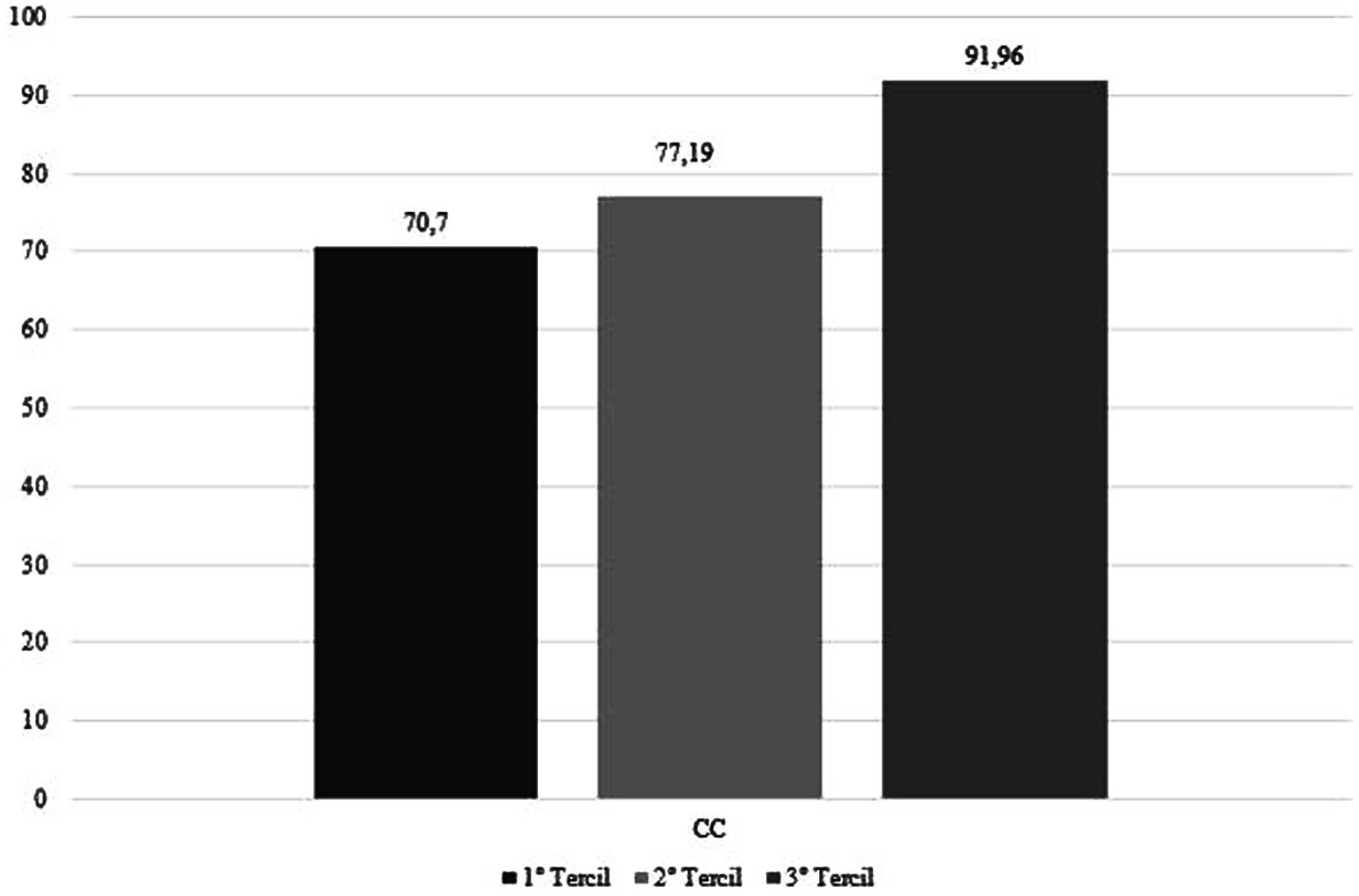

Figura 4 - Variação das médias de CC (cm) conforme tercis de BRI, Jaguaruana-Ceará, 2017. BRI = body roundness index; CC = circunferência da cintura. BRASPEN J 2020; 35 (4): 392-401 


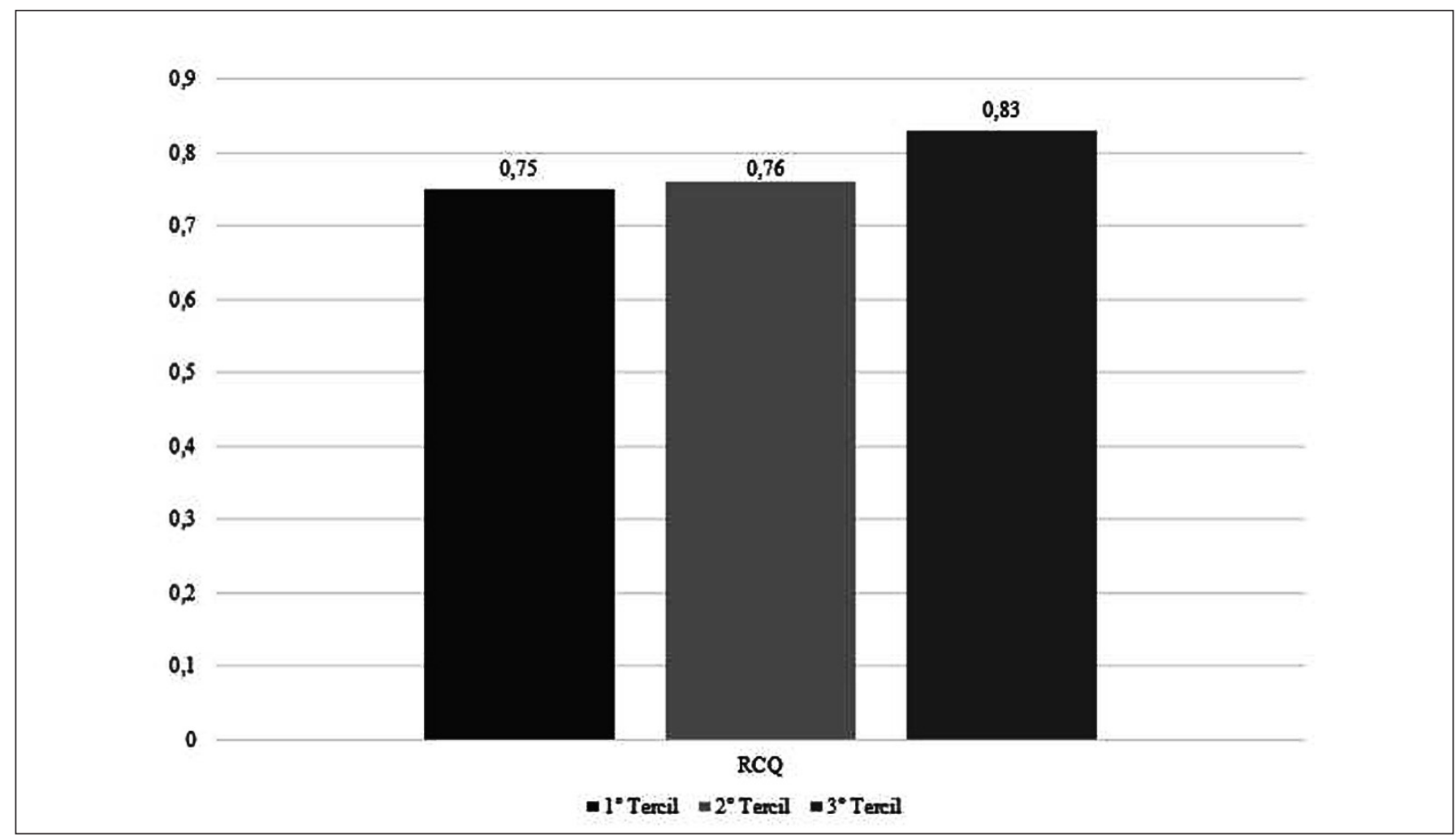

Figura 5 - Variação das médias de RCQ conforme tercis de BRI, Jaguaruana-Ceará, 2017. BRI = body roundness index; $R C Q=$ relação cintura-quadril.

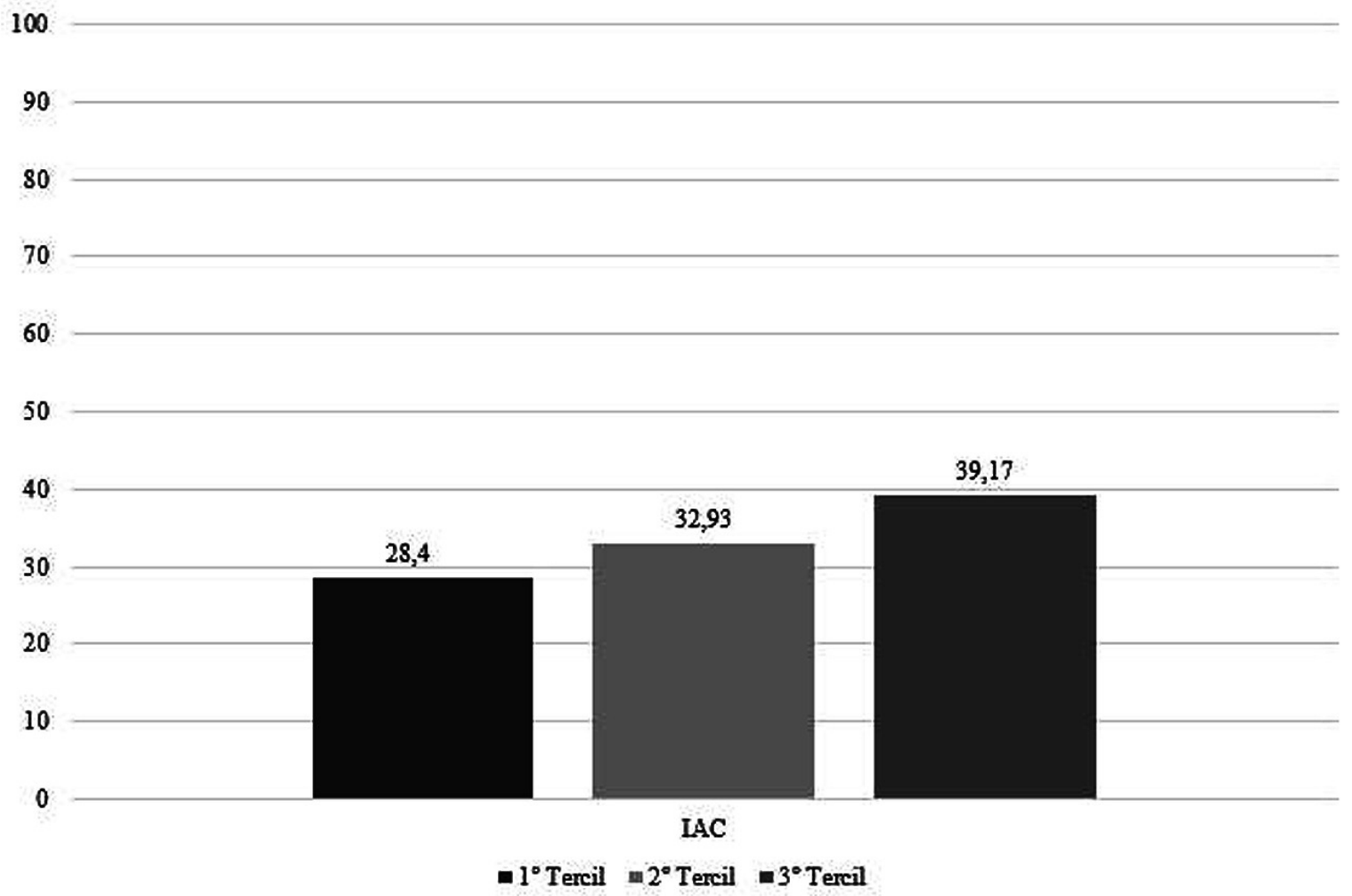

Figura 6 - Variação das médias de IAC (\%) conforme tercis de BRI, Jaguaruana-Ceará, 2017. BRI = body roundness index; IAC = indice de adiposidade corporal. 


\section{DISCUSSÃO}

Observou-se, em relação ao estado nutricional, que as participantes apresentaram um diagnóstico predominante de eutrofia, mas isto apenas porque o diagnóstico de obesidade foi estratificado, pois ao somar as porcentagens de sobrepeso e obesidade observa-se que $55 \%$ das pacientes encontraram-se na condição de excesso de peso, segundo - IMC. Já para o IAC, 50\% das participantes apresentaram quantidade de gordura corporal normal e as demais (50\%), excesso compatível com sobrepeso ou obesidade. Quanto à análise de aumento de médias entre esses índices, o ABSI comportou-se de maneira diretamente proporcional com $R C Q$ e o BRI com IMC, CC, RCQ e IAC.

As mulheres também apresentaram várias doenças comumente associadas à SOP, como HAS e cálculos renais, o que também pôde ser visto em outros estudos ${ }^{12}$. Adicionado a essas, por estar a resistência insulina na base etiológica da síndrome, uma variedade de outras comorbidades podem ser desenvolvidas, com destaque para o diabetes mellitus tipo 2 (DM2). A predisposição para esta desordem metabólica aumenta neste público, em especial se há sobrepeso ou obesidade, prática insuficiente de exercícios físicos e maus hábitos alimentares ${ }^{13}$.

As DCV também são comumente associadas à SOP, seus mecanismos fisiopatológicos ainda não são completamente elucidados, mas sabe-se que a adiposidade visceral favorece esse quadro ${ }^{10}$. Em estudo recente observou-se que o uso de anticoncepcionais, tratamento mais comum, exerce efeito pró-trombótico sobre as mulheres, o que em combinação com a resistência à ação da insulina pode desencadear DCV ${ }^{14}$. No presente estudo, o diagnóstico de HAS foi referido por 7,5\% das mulheres, prevalência que está entre os achados clínicos de outras pesquisas realizadas com mulheres com SOP, que evidenciaram percentuais de $5,8 \%$ e $10 \%{ }^{15,16}$.

Percebeu-se que $45 \%$ das participantes encontravam-se em tratamento para a SOP, todas por meio de anticoncepcionais, que ainda é a principal estratégia terapêutica utilizada. Entretanto, a literatura vem mostrando que o uso de anticoncepcionais não é o método mais eficaz para tratar a doença, visto que eles melhoram a anovulação, mas não a base da doença, que é a resistência à ação da insulina, e ainda podem agravar esse quadro ${ }^{14,17}$. Assim, vêm sendo propostas novas abordagens terapêuticas focadas na melhoria da resistência à insulina, dentre as quais está a reeducação alimentar, com dieta equilibrada, hipocalórica e de baixo índice glicêmico, prática regular e orientada de exercícios físicos, controle medicamentoso da insulina com drogas e gerenciamento do estresse ${ }^{18}$.

Como uma característica bastante presente na SOP, este estudo apontou que a maior parte das mulheres (55\%) sofre de amenorreia, conforme corroborado previamente por outros autores $^{19}$, os quais atribuem o distúrbio à hiperinsulinemia que estimula a secreção androgênica pelos ovários e adrenais e suprime a produção hepática da globulina transportadora dos hormônios sexuais, com aumento dos androgênios livres biologicamente ativos. $\bigcirc$ excesso local dos androgênios ovarianos causa a atresia prematura dos folículos ovarianos, formando pequenos cistos e anovulação crônica. $\bigcirc$ estado nutricional mostrou-se adequado no grupo, visto que $40 \%$ apresentaram eutrofia, segundo o IMC, 57,5\% não demonstraram CC de risco para complicações metabólicas, 82,5\% não apresentaram riscos para $D C V$, segundo a RCQ e $50 \%$ foi classificada com adiposidade normal, segundo o IAC.

Entretanto, quando analisadas as médias de índices/ medidas do grupo, notou-se que o IMC médio sugeriu préobesidade, a CC média correspondeu a risco elevado para complicações metabólicas e o IAC teve média condizente com sobrepeso. Isto sugere que, embora a maior parte das mulheres tenha estado dentro da normalidade quanto ao diagnóstico nutricional, aquelas que foram classificadas com parâmetros acima do adequado tiveram excessos tão significativos, que foram capazes de elevar acentuadamente as médias de cada índice. Portanto, intervenção nutricional sobre essa parcela do grupo mostra-se necessária.

Um estudo realizado no Maranhão evidenciou achados opostos, uma vez que a maioria das mulheres com SOP apresentou sobrepeso pelo IMC (60\%), risco para complicações metabólicas segundo a CC (54\%) e risco para doenças cardiovasculares conforme a RCQ $(51,4 \%)^{20}$. Em outro trabalho realizado em Minas Gerais, encontrou-se que $96,3 \%$ das mulheres apresentavam excesso de peso, $90,7 \%$, risco para complicações metabólicas e 74,1\%, risco para $\mathrm{DCV}^{2}$.

Sobre os índices antropométricos mais recentes, viu-se que não foram sugestivos de risco metabólico, uma vez que a maior parte das participantes esteve abaixo das médias do $\mathrm{ABSI}(52,5 \%)$ e do BRI (95\%), além deste último ter sugerido uma forma corporal levemente arredondada. Também, percebeu-se correlação positiva estatisticamente significante de ABSI com RCQ e de BRI com IMC, CC, $R C Q$ e IAC.

Sabe-se que ABSI e BRI ainda são índices antropométricos pouco explorados pela literatura, mas alguns estudos já lançaram mão destes marcadores para explorar e classificar a composição corpórea e risco de mortalidade de populações. Em um estudo realizado na Polônia, que avaliou o $A B S I$ versus IMC como parâmetros de avaliação antropométrica e de risco de saúde, em homens jovens sedentários, constatou-se que o $\mathrm{ABSI}$ se correlacionou melhor que o IMC com os níveis de triacilgliceróis 
circulantes e também com a resistência insulínica, sendo assim, um melhor preditor que o IMC para indicar distúrbios metabólicos ${ }^{21}$.

Já em outro estudo, realizado em Nova York, que avaliou a concordância entre IMC e ABSI no diagnóstico antropométrico de indivíduos saudáveis, constatou-se que o $\mathrm{ABSI}$, por quantificar a forma corporal de uma forma que o IMC não avalia, isto é, com foco na adiposidade abdominal, serve como melhor parâmetro para predizer risco de comorbidades metabólicas e mortalidade ${ }^{22}$. Contudo, também foi sugerido que a combinação entre $A B S I, I M C, C C$ e RCQ representa uma estratégia mais adequada na prática clínica para avaliar os pacientes do que estes índices/medidas isoladamente. Em outro estudo recente realizado na cidade de Tokyo, observou-se que o $A B S I$, por ser uma medida indireta de adiposidade abdominal, foi associado à rigidez arterial em pacientes com $\mathrm{DM} 2^{23}$.

Pôde se constatar pelo presente estudo que o ABSI é um bom índice para mensurar a adiposidade abdominal, então infere-se que talvez por isto ele se correlacione positivamente com a RCQ e não com outros índices, como IMC e IAC, que mensuram adiposidade corporal total, sem distinção de localização ou forma.

Com relação ao BRI, um estudo desenvolvido nos Estados Unidos evidenciou que este índice prediz satisfatoriamente os percentuais de gordura corporal e tecido adiposo total ${ }^{9}$. Também, percebeu-se que BRI e IMC correlacionaram-se positivamente, mas que BRI é mais adequado que IMC, uma vez que é proporcional aos valores de gordura visceral e não somente avalia a relação peso/altura.

Um estudo realizado no Norte do Irã, que investigou a associação entre $B R I, A B S I, C C, R C Q$ e o diagnóstico de doença hepática gordurosa não alcoólica (DHGNA), concluiu que BRI e CC são mais fortemente correlacionados com o diagnóstico de DHGNA do que $A B S I$ e $R C Q$, reforçando o potencial do BRI em prever o surgimento de doenças hepáticas, cardíacas, hiperuricemia e diabetes ${ }^{24}$.

Outro estudo realizado na Holanda investigou a sensibilidade de BRI e ABSI para identificar fatores de risco para DCV em indivíduos adultos ${ }^{25}$. Percebeu-se que o BRI é válido para predizer fatores de risco para DCV por meio do modelamento matemático do corpo, mas não superior a medidas como IMC e CC. Já o ABSI não foi eficiente em identificar fatores de risco para DCV.

A correlação positiva do BRI com uma variedade maior de índices/medidas tradicionais talvez se deva ao fato deste índice avaliar matematicamente a circularidade corporal total, utilizando-se de medidas que não priorizam a adiposidade localizada.

Portanto, os achados na literatura têm mostrado que estes índices mais atuais parecem predizer adequadamente desordens metabólicas e devem ser valorizados em uma avaliação seriada, que possibilite comparações dessas medidas ao longo do tempo.

Um estudo realizado na China, que teve como objetivo avaliar a capacidade do ABSI e do BRI em identificar indivíduos em risco para DM2, apontou que nem ABSI nem BRI foram melhores que os índices/medidas tradicionais (IMC, $C C, R C Q)^{26}$. O ABSI se mostrou mais fraco preditor que o $B R I$, enquanto medida alternativa para avaliar o risco ou presença de DM2, sugerindo que, apesar de tantos estudos favoráveis, sejam desenvolvidas novas pesquisas para avaliar as vantagens e desvantagens destes índices antropométricos mais recentemente propostos.

Por fim, visando estimular que mais estudos sejam realizados com portadoras de SOP, com o intuito de contribuir com a melhoria do estado nutricional e reduzir suas comorbidades, explicita-se aqui o pequeno número amostral como limitação deste estudo e que precisa ser considerada em estudos posteriores, bem como sugere-se que mais pesquisas sejam realizadas, agora avaliando o consumo alimentar dessas mulheres, identificando quais componentes dele podem ser contribuintes para a resistência à insulina e intervindo naquelas diagnosticadas com estado nutricional inadequado e consumo alimentar favorecedor da resistência à insulina.

O estudo apresentou algumas limitações, como o pequeno tamanho amostral, o que se deveu à recusa em participar por parte da população elegível. Entretanto, este aspecto não impossibilitou o achado de resultados estatisticamente significantes. Ainda, não foi realizada a avaliação da gordura corporal a partir de métodos reconhecidamente precisos, como a bioimpedância, contudo, lançou-se mão do $A B S I$ e BRI, índices antropométricos mais atuais, cujas estimativas têm mostrado forte associação com risco de doenças crônicas.

\section{CONCLUSÃO}

As participantes portadoras de SOP apresentaram predomínio de algum grau de excesso de peso em relação ao $I M C$ e ausência de risco para DCV quanto à $C C$ e à $R C Q$. Pelo IAC, metade das participantes foi diagnosticada com excesso de adiposidade corporal. Constatou-se, ainda, que as participantes nos maiores tercis de $A B S I$ apresentaram também maiores valores de $R C Q$, o mesmo comportamento diretamente proporcional, sendo observado para o BRI em relação a todos os índices/medidas tradicionais.

A combinação dos índices/medidas antropométricas tradicionais com os mais recentemente propostos parece possibilitar uma melhor e mais completa compreensão acerca do estado nutricional, em especial se realizadas de forma seriada. 


\section{REFERÊNCIAS}

1. Escobar-Morreale HF. Polycystic ovary syndrome: definition, aetiology, diagnosis and treatment. Nat Rev Endocrinol. 2018;14(5):270-84.

2. Calixto CFS, Paula TMD, Cândido AL, Rodrigues AMS, Santos LC, Ferreira AVM, et al. Estado nutricional e consumo alimentar de pacientes portadoras de síndrome de ovários policísticos. Rev Min Enferm. 2012;16(2):159-65.

3. Barber TM, Hanson P, Weickert MO, Franks S. Obesity and polycystic ovary syndrome: implications for pathogenesis and novel management strategies. Clin Med Insights Reprod Health. 2019;13:1179558119874042.

4. Chitme HR, Al Azawi EAK, Al Abri AM, Al Busaidi BM, Salam ZKA, Al Taie MM, et al. Anthropometric and body composition analysis of infertile women with polycystic ovary syndrome. J Taibah Univ Med Sci. 2017;12(2):139-45.

5. World Health Organization (WHO). EHO recommendations on adolescent sexual and reproductive health and rights. Geneva: WHO; 2018.

6. Flegal KM, Ioannidis JPA, Doehner W. Flawed methods and inappropriate conclusions for health policy on overweight and obesity: the Global BMI Mortality Collaboration meta-analysis. J Cachexia Sarcopenia Muscle. 2019;10(1):9-13.

7. World Health Organization. Obesity: preventing and managing the global epidemic. Report of a World Health Organization Consultation. WHO Obesity Technical Report Series, n. 284. Geneva: World Health Organization; 2000. p.256.

8. Bergman RN, Stefanovski D, Buchanan TA, Sumner AE, Reynolds JC, Sebring NG, et al. A better index of body adiposity. Obesity (Silver Spring). 2011;19(5):1083-9.

9. He S, Chen X. Could the new body shape index predict the new onset of diabetes mellitus in the Chinese population? PLoS One. 2013;8(1):e50573.

10. Thomas DM, Bredlau C, Bosy-Westphal A, Mueller M, Shen W, Gallagher D, et al. Relationships between body roundness with body fat and visceral adipose tissue emerging from a new geometrical model. Obesity (Silver Spring). 2013;21(11):2264-71.

11. Krakauer NY, Krakauer JC. A New body shape index predicts mortality hazard independently of body mass index. Plos One. 2012;7(7):e39504.

12. Fedrigon D $3^{\text {rd }}$, Alazem K, Sivalingam S, Monga M, Calle J. Nephrolithiasis and polycystic ovary syndrome: a case-control study evaluating testosterone and urinary stone metabolic panels. Adv Urol. 2019;2019:3679493.

13. Polakl K, Czyzyk A, Simoncini T, Meczekalski B. New markers of insulin resistance in polycystic ovary syndrome. J Endocrinol Invest. 2017;40(1):1-8.
14. Chiharu Iwata M, Porquere L, Sorpreso IC, Baracat EC, Soares Júnior JM. Association of oral contraceptive and metformin did not improve insulin resistance in women with polycystic ovary syndrome. Rev Assoc Med Bras (1992). 2015;61(3):215-9.

15. Michalak R, Jagodzinska A, Zieleniewski W. The prevalence of high blood pressure as one of cardiovascular risk factors among women with polycystic ovary syndrome. Arterial Hypertension. 2015;19(1):19-22.

16. Joham AE, Boyle JA, Zoungas S, Teede HJ. Hypertension in reproductive-aged women with polycystic ovary syndrome and association with obesity. Am J Hypertens. 2015;28(7):847-51.

17. Amato MC, Vesco R, Vigneri E, Ciresi A, Giordano C. Hyperinsulinism and polycystic ovary syndrome (PCOS): role of insulin clearance. J Endocrinol Invest. 2015;38(12):1319-26.

18. Jarret BY, Lujan ME. Impact of hypocaloric dietary intervention on ovulation in obese women with PCOS. Reproduction. 2016;REP-16-0385.

19. Yilmaz SA, Kerimoglu OS, Pekin AT, Incesu F, Dogan NU, Celik $\mathrm{C}$, et al. Metastin levels in relation with hormonal and metabolic profile in patients with polycystic ovary syndrome. Eur J Obstet Gynecol Reprod Biol. 2014;180:56-60.

20. Fonsêca PCA, Carvalho CA, Sousa RML, Brito LMO, Chein MBC. Estado nutricional e risco cardiovascular de mulheres portadoras de síndrome dos ovários policísticos. Rev Pesq Saúde. 2011;12(1):47-51.

21. Malara M, Keska A, Tkaczyk J, Lutoslawka G. Body shape index versus body mass index as correlates of health risk in young healthy sedentary men. J Transl Med. 2015;13:75.

22. Krakauer JC, Krakauer NY. Combining body mass and shape indices in clinical practice. Case Rep Med. 2016;2016:1526175.

23. Bouchi R, Asakawa M, Ohara N, Nakano Y, Takeuchi T, Murakami $M$, et al. Indirect measure of visceral adiposity 'A Body Shape Index' (ABSI) is associated with arterial stiffness in patients with type 2 diabetes. BMJ Open Diabetes Res Care. 2016;4(1):e000188.

24. Motamed N, Rabiee B, Hemasi GR, Ajdarkosh H, Khonsari MR, Maadi M, et al. Body roundness index and waist-to-height ratio are strongly associated with non-alcoholic fatty liver disease: a population-based study. Hepat Mon. 2016;16(9):e39575.

25. Maessen MF, Eijsvogels TM, Verheggen RJ, Hopman MT, Verbeek AL, de Vegt F. Entering a new era of body indices: the feasibility of a body shape index and body roundness index to identify cardiovascular health status. PLoS One. 2014;9(9):e107212.

26. Chang Y, Guo X, Chen Y, Guo L, Li Z, Yu S, et al. A body shape index and body roundness index: two new body indices to identify diabetes mellitus among rural populations in northeast China. BMC Public Health. 2015;15:794.

Local de realização do estudo: Unidade Básica de Saúde da Família Dr. Carlos Alberto de Araújo (Sede I), Jaguaruana, CE, Brasil.

Conflito de interesse: Os autores declaram não haver. 